INTERIM REPORT

U.S. Department of Energy

\title{
PLANT RHIZOSPHERE EFFECTS ON METAL MOBILIZATION AND TRANSPORT
}

\author{
Principal Investigator: Teresa W-M. Fan \\ University of California Davis \\ Coinvestigator: Richard M. Higashi and David E. Crowley \\ Project Number: 55118 \\ Grant Number: DE-FG07-96ER20255 \\ Grant Project Officer: James Tavares \\ Project Duration: September 1997 - August 2000
}

\section{EXECUTIVE SUMMARY}

The myriad of human activities including strategic and energy development at various DOE installations have resulted in the contamination of soils and waterways that can seriously threaten human and ecosystem health. Development of efficacious and economical remediation technologies is needed to ameliorate these immensely costly problems. Bioremediation (both plant and microbe-based) has promising potential to meet this demand but still requires advances in fundamental knowledge. For bioremediation of heavy metals, the three-way interaction of plant root, microbial community, and soil organic matter (SOM) in the rhizosphere is critically important for long-term sustainability but often underconsidered. Particularly urgent is the need to understand processes that lead to metal ion stabilization in soils, which is crucial to all of the goals of bioremediation: removal, stabilization, and transformation. We have developed the tools for probing the chemistry of plant rhizosphere and generated information regarding the role of root exudation and metabolism for metal mobilization and sequestration.

\section{Relevance, Impact, and Technology Transfer}

In the long run, this research should lead to fundamental advances in the understanding of plant-microbe interactions and how these interactions govern the long-term fate of heavy metals in belowground ecosystems. There should be direct applicability of these tools and knowledge to facilitate and enhance field implementation and monitoring of metal bioremediation.

\section{METHODS AND RESULTS}

Our work has focused on examining the influence of important rhizosphere factors (i.e. root exudation chemistry and SOM) on metal ion accumulation and metabolism in crop plants such as barley, wheat, and rice. In order to understand the chemistry involved in metal ion mobilization by plants, we developed advanced NMR methodology in combination with GC-MS for a comprehensive profiling of root exudates (Fan et al., 1997a; Fan et al., 1999; Fan and Lane, in press), which has been a difficult challenge by conventional approach. Using a similar approach, the intracellular profiles of metal reactive compounds (MRC) in plants were also acquired, which is important to the understanding of how metal ions are sequestered within plants. Moreover, we have completed the development of a rapid and parallel sample analysis (gel electrophoretic) method for characterizing tissue thiol-rich peptides (important to sequestration of $\mathrm{Cd}$ and other heavy metals) (Fan et al., 1999). 
These methodologies have been applied to investigate MRC production and its role in metal ion accumulation as a function of plant species, genotypes, transition metals, Cd, as well as SOM treatments. The main set of experiments focussed on wheat since a full set (7) of Chinese Spring (CS) genotypes (each with one chromosome addition from the salt and Al-tolerant European saltgrass, Lophopyrum sp.) are available from Dr. J. Dvorak, UC Davis, from a project funded by DOE. Utilizing these and other recombinant wheat genotypes, functional genomic maps are now being generated by Dr. Dvorak, now as part of an NSF-funded functional genomic center.

The root exudate profiles differed both qualitatively and quantitatively among barley, CS wheat, and rice plants (Figure 3). Under Fe deficiency, the family of mugineic acid (MA) phytosiderophores (PS) were among major components in barley and wheat exudates but was below NMR detection in rice exudate. The barley and wheat exudates were also generally higher in MRC (e.g. amino and organic acids) concentrations. These differences are related to a better ability of barley and wheat to extract Fe than rice plant.

The increase in exudation of mugineic acids (MAs) under Fe deficiency in barley (3-epihydroxy-MA, MA, and 2'-DMA) and CS wheat (2'-deoxymugineic acid, 2'-DMA) is consistent with their role in Fe acquisition. The MAs may also be involved in the accumulation of $\mathrm{Cu}, \mathrm{Zn}$, and $\mathrm{Mn}$. However, the MAs exudation were poorly correlated with $\mathrm{Cd}$ acquisition by either barley or wheat plants. This is contrary to the general notion that PS may mediate heavy metal uptake by plants. Instead, independent of root MRC exudation, the $\mathrm{Cd}$ treatment caused a large accumulation of transition metals in wheat (Fan et al., 2000a \& 2001; Shenker et al., submitted to JEQ). This Cd-transition metal intereffect is not generally known and we presently suspect is due to a disrupting effect of $\mathrm{Cd}$ on root membrane and/or exudation of extracellular polysaccharides (EPS). It is also interesting to note that despite the absence of any added $\mathrm{Sr}$ salts, a large Sr accumulation was observed as a result of Sr impurity in added nutrient salts.

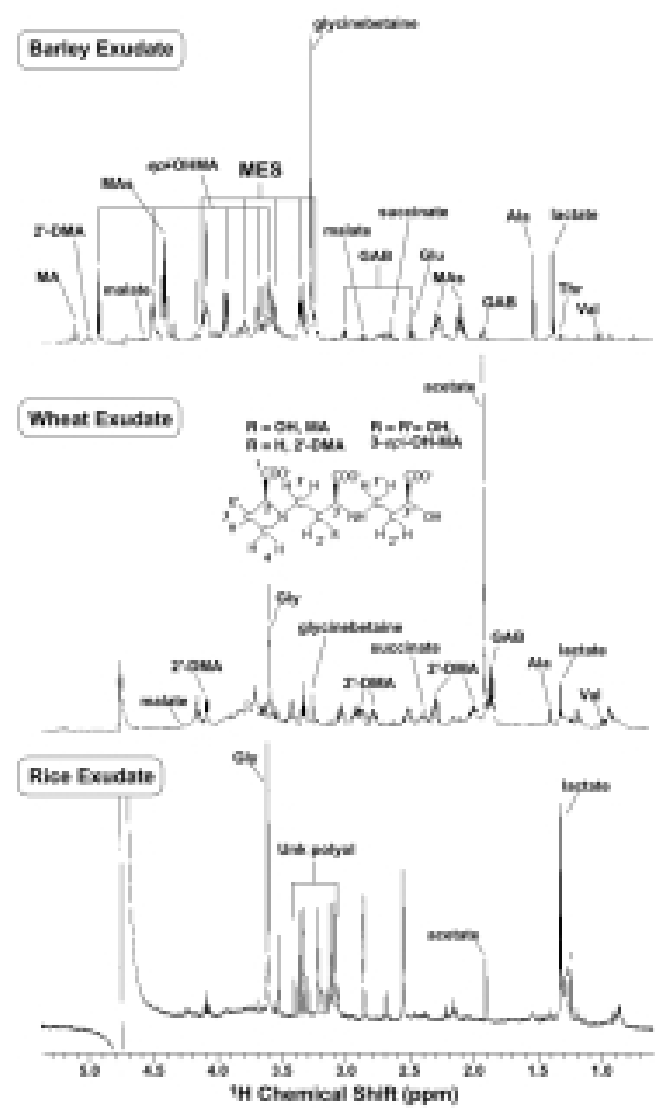

Figure 3. ${ }^{1} \mathrm{H}$ NMR spectra of whole root exudates from barley, wheat, and rice under a combined $\mathrm{Fe}$ deficient and $\mathrm{Cd}$ treatment. GAB, $\gamma$-amino-butyrate; MES, morpholino-ethanesulfonate; epi-OHMA, epihydroxy mugineic acid; 2'-DMA, 2'-deoxy-mugineic acid; Unk polyol, unknown polyol

In addition to root exudation, the role of SOM (e,g, soil HS) in heavy metal accumulation by plants are being investigated. Background information is grossly lacking in this area, but necessary for understanding heavy metal mobilization by plants from soils. The experiments to date indicates that $\mathrm{HS}+\mathrm{Cd}$ treatment of $\mathrm{CS}$ wheat plants did not attenuate, and even slightly increased the accumulation of some transition metals and $\mathrm{Cd}$ in roots, which is contrary to HS role as a competitive chelator for metal ions (Figure 4). Despite this enhanced metal accumulation, the presence of HS stimulated plant growth, improved MRC production, and alleviated part of the Cd-induced growth inhibition. It is likely that some HS component(s) may directly mediate metal ion (e.g. $\mathrm{Cd}, \mathrm{Zn}, \mathrm{Ni}$ ) uptake via their cotransport with metal 
ions into wheat roots. These metal ions may be stabilized by enhanced MRC (amino acids, organic acids, and PC) accumulation in wheat roots and shoots. The observed release of substances chemically identifiable as HS back into the root exudate is consistent with HS uptake. It also agrees with the high affinity of HS for Cd(II), as observed by py-GCMS (Higashi et al., 1998).
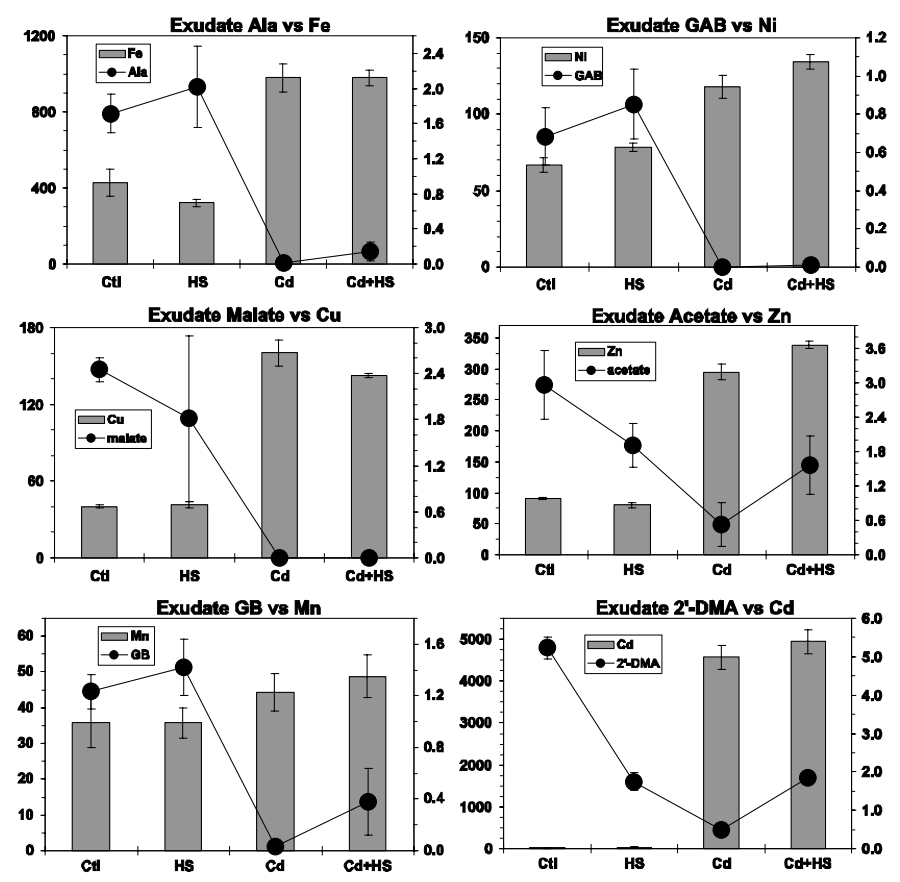

Figure 4. Comparison of exudate MRC concentration versus metal content in wheat roots under a combination of soil humate (HS, 5 ppm) and $\mathrm{Cd}(5 \mathrm{ppm})$ treatments. All MRCs declined drastically in exudation while accumulation of transition metals in roots was elevated with $\mathrm{Cd}$ or $\mathrm{Cd}+\mathrm{HS}$ treatments. The presence of $5 \mathrm{ppm} \mathrm{Cd}$ in growth media resulted in a nearly $1000-$ fold accumulation in wheat roots.

In view of the importance of HS in metal ion sequestration in soils and intimate connection to plant-microbe interactions, we have devoted part of our effort in developing concerted measurements by py-GCMS, NMR, FT-IR, and other biophysical methodologies for characterizing molecular structure motifs of HS that are likely to be involved in metal ion interaction (Fan et al., 2000b; Fan and Lane, in press). In addition, by combining natural abundance carbon isotope ratio (CIR) measurement with py-GCMS, we are developing the ability to track the turnover kinetics of various HS structural fragments based on their $\delta^{13} \mathrm{C}$ (e.g. Figure 5). This capability will open the door for probing the chemical mechanism of humification and reveal those substructures of HS that become recalcitrant to turnover by interacting with metal ions. This knowledge is required for designing and implementing metal remediation via both stabilization and mobilization strategies.

Moreover, the impact of metals on microbial communities, sensitive species in particular, was investigated. The toxic effects of $\mathrm{Cd}$ on substrate versatility and metabolic redundancy of soil microbial communities were analyzed using PCR-DGGE (Wang et al., submitted to Appl. Environ. Microbiol.). In a screening assay examining the numbers of different bacterial species that grew on 30 selected carbon substrates, sensitive species were lost at $5 \mathrm{ppm} \mathrm{Cd}$ with further losses occurring at increasing levels of contamination. This species loss was associated with a decline in the rate of substrate utilization, and in several instances with complete failure to utilize certain substrates in a short term assay. Based on these results, we predict that heavy metal loading of plant tissues will significantly affect the composition and activity of the microbial communities that degrade plant litter and synthesize soil organic matter. 
We have also begun to characterize the CS wheat genotypes plus the parent for their difference in capacity of metal accumulation and root exudation profile. Analysis to date indicates that the genotypic profile of $\mathrm{Cd}$ accumulation differed from those of the transition metals and that the metal accumulation system in wheat may reside on at least two chromosomal loci (Fan et al., 2000a).

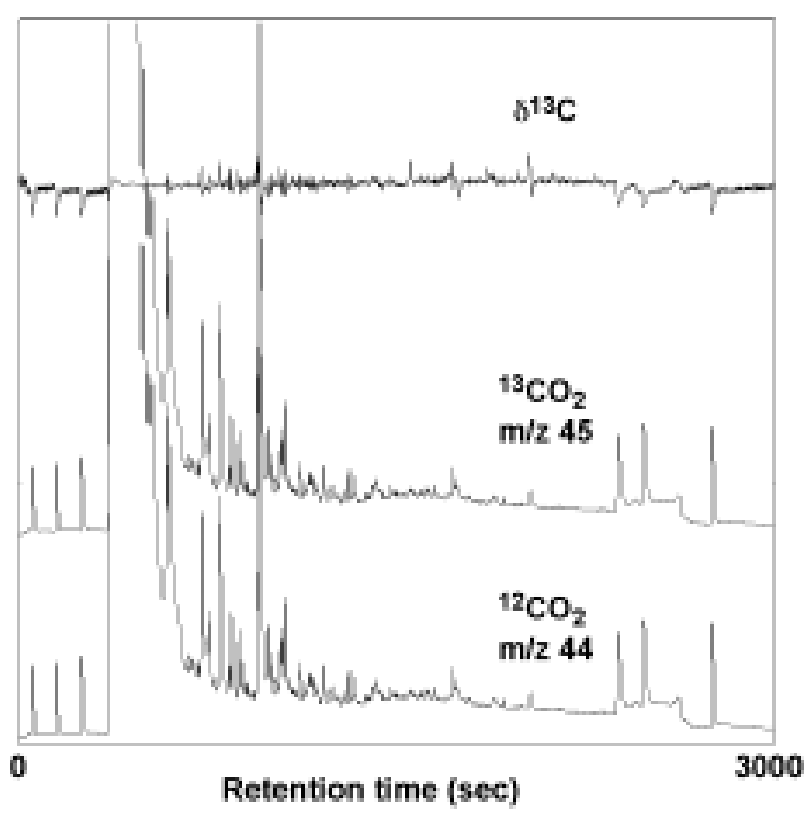

Figure 5. Pyrolysis-GC-CIR-MS analysis of soil beneath plants flushed with ${ }^{12} \mathrm{C}$-enriched $\mathrm{CO}_{2}$. Pyrolysis is performed in inert helium gas, so it is distinct from combustion analysis The three channels $-{ }^{12} \mathrm{C}$ channel, ${ }^{13} \mathrm{C}$ channel, and ratio $\left(\delta^{13} \mathrm{C}\right)$ - are shown on their own ordinate scales and offset for illustrative purposes. On the ratio $\left(\delta^{13} \mathrm{C}\right)$ channel, it is clear that some peaks are $(+)$, and some are (-) relative to an arbitrary null line. The sharp trio of pulses at the beginning and end of the chromatogram (seen as negative peaks in the ratio channel) are reference $\mathrm{CO}_{2}$ infusions, which provide data for properly scaling the sample $\delta^{13} \mathrm{C}$. The data shown here is only for illustration of the method; for precise quantification of $\delta^{13} \mathrm{C}$, minor data adjustments are needed. (Our thanks to Dr. D. Harris for codevelopment of the method, and to Dr. K. van Kessel for the sample).

The MRC involved in intracellular sequestration of $\mathrm{Cd}(\mathrm{II})$ was investigated using the recently developed method that combined fluorescent tagging of thiol-rich peptides by bromobimane with SDS-PAGE. This method allowed a fast and simultaneous assay of both phytochelatins (PC) and metallothionein-like proteins, as illustrated for wheat, rice, and clam in Figure 6. For both wheat and rice, $\mathrm{Cd}$ treatments induced a large accumulation of $\mathrm{PC}(<3.5 \mathrm{kD}$ $\mathrm{SH}-$ rich peptides) but not any other SH-rich proteins in root and shoot. The PC accumulation was sensitive to $\mathrm{Cd}$ accumulation only. These peptides exhibited a high affinity for $\mathrm{Cd}$ and $\mathrm{Pb}$, as revealed by chelator competition and capillary electrophoresis coupled with ICP-MS (in collaboration with Dr. V. Majidi, Los Alamos National Lab.). These results suggests PC's role in sequestering heavy metals, particularly in roots where much higher amounts of Cd were accumulated than in shoots. As part of the plant root litter, the fate of these metal-PC complexes in soils will need to be understood for both metal stabilization or remobilization.

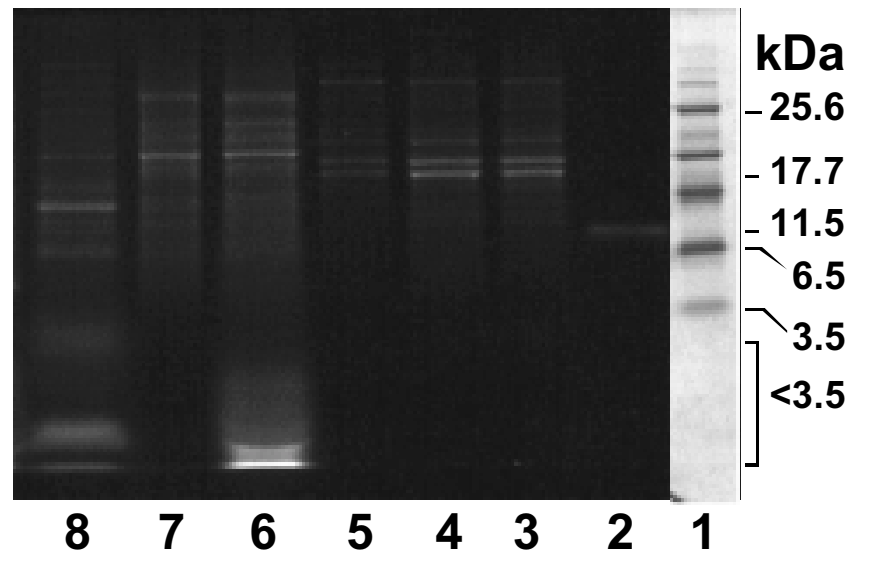

Figure 6. SDS-PAGE of bromobimanetagged SH-rich peptides and proteins from wheat, rice, and clam tissues. Lanes 1, MW standards; 2, rabbit metallothionein (MT); 3-5, clam MT extracts; 6, Cd-treated and 7, control wheat root extracts; 8, Cd-treated rice shoots. 
In conclusion, our results indicate that both SH-rich peptides and non-peptidic MRC may be important to heavy metal accumulation in plant tissues. They may all contribute towards plant's ability to tolerate high levels of metal accumulation and may influence subsequent fate of metals in the humification process. In addition, plant roots accumulated a high amount of metals, which did not appear to be mediated by the exudation of phytosiderophores or small water-soluble MRC. This raises the question on the role of macromolecular components in the rhizosphere (e.g. HS, EPS) in mediating heavy metal uptake. Moreover, the metals sequestered by roots will not be practical to remove from soils by simple harvest. From the phytoremediation standpoint, the long-term fate of these metals including bioavailability and leachability will need to be addressed. Depending on their speciation (e.g. formation of PC complexes) and interaction with soil organic matter and minerals, pollutant metals may be "entombed" with aging (Cunningham and Ow, 1996). Such aging process, if understood, may be enhanced to stabilize metal ion movement and transport in the subsurface zone.

\section{Personnel Supported}

Facu.lty (total of 6):

Richard M. Higashi, David Crowley, Teresa W.-M. Fan, Andrew N. Lane, John Bartley, Vahid Majidi

Postoctoral (total of 3)

Judy Pedler, Moshe Shenker, Fabienne Baraud

Graduate student (1)

Robert Kaufman

\section{Manuscript Published and Submitted}

Shenker, M., T.W.-M. Fan, and D.E. Crowley, Phytosiderophores Influence on Cadmium Uptake by Wheat and Barley Plants (submitted to Journal of Environmental Quality).

Fan, T.W.-M. and A.N. Lane, NMR in the Plant-Soil Environment, Encyclopedia of Analytical Chemistry, John Wiley and Sons, New York, (in press).

Fan, T.W.-M, Lane, A.N., Shenker, M., Bartley, J.P., Crowley, D. and Higashi, R.M. Comprehensive chemical profiling of gramineous plant root exudates using high-resolution NMR and MS. Phytochem. 57, 209-221 (2001).

Fan, T.W.-M., Baraud, F., Higashi, R.M., eds. Eller, P.G. and Heineman, W.R. Genotypic influence on metal ion mobilization and sequestration via metal ion ligand production by wheat. In: Nuclear Site Remediation. American Chemical Society Symposium Series, vol. 778, pp. 417-431, Washington, D.C (2000a).

Fan, T. W.-M., R. M. Higashi and A. N. Lanes, Chemical Characterization of a Chelator-Treated Soil Humate by Solution-State Multinuclear Two-Dimensional NMR with FTIR and Pyrolysis-GCMS, Environmental Science and Technology 34, 1636-1646 (2000b).

Higashi, R.M., T.W.-M. Fan, A.N. Lan, Association of desferrioxamine with humic substances and their interaction with cadmium(II) as studied by pyrolysis gas chromatography mass spectrometry and nuclear magnetic resonance spectroscopy, Analyst 123(5), 911-918 (1998).

Fan, T.W.-M., J. Pedler, A.N. Lane, D. Crowley, and R.M. Higashi, Comprehensive Analysis of Organic Ligands in Whole Root Exudates Using NMR and GC-MS, Analytical Biochemistry 251, 57-68 (1997). 


\section{Interactions}

a. Meetings Participated

"A Rapid and Simultaneous Electrophoretic Assay for Phytochelatin And Metallothionein Induction in Plants And Aquatic Invertebrates", Teresa W.-M. Fan, Robert Kaufman, Vahid Majidi, and Richard M. Higashi, PacifiChem meeting of American Chemical Society, Honolulu (Dec., 2000)

"Interactions of Metal Ions with Biogenic Ligands, Complex Inorganic Mixtures, and Humic Substances That Affect Bioavailability ff Metal Ions to Plants", Richard M. Higashi, Teresa W-M. Fan, and Andrew N. Lane, PacifiChem meeting of American Chemical Society, Honolulu (Dec., 2000)

"Heavy Metal Bioavailability and Sequestration by Plants via Interaction of Plant Root Exudate and Soil Humic Ligands", Teresa W-M Fan, Fabienne Baraud, and Richard Higashi, Annual Meeting of American Chemical Society, Washington, DC (Aug., 2000)

"Characterization of Peptidic Structures in A Chelator-Treated Soil Humate by Solution-State Multinuclear Two-Dimensional NMR with FTIR and Pyrolysis-GCMS", Teresa W-M Fan, Richard Higashi, and Andrew N. Lane, Annual Meeting of American Chemical Society, Washington, DC (Aug., 2000)

"Interaction between Contaminant Metal Mobility and Soil Fertility Manipulated by Organic Amendments", Teresa James, Teresa W.-M. Fan, and Richard M. Higashi, Annual Meeting of American Chemical Society, Washington, DC (Aug., 2000)

"Probing the Characteristics and Properties of Humic Substances That Affect the Bioavailability of Cd(II) to Plants and Phenathrene to Microbes", Richard M. Higashi, Teresa W-M. Fan, EPA/DOE/ONR/NSF Joint Bioremediation Workshop, Chicago (Nov., 1999)

"Influence of Soil Organic Matter Composition on Desorption and Biodegradation of Aromatic Pollutants", T.M. Young, K.M. Scow, R.M. Higashi, T.W-M. Fan, E. Schwartz, L.F. Schultz, N. Watanabe, and S. Loebmann, EPA/DOE/ONR/NSF Joint Bioremediation Workshop, Chicago (Nov., 1999).

"Interaction of Heavy Metal Sequestration and Production of Metal Ion Ligands in Wheat under Fe Deficient, Cd, and Soil Humic Treatments", Teresa W-M Fan, Fabienne Baraud, and Richard Higashi, EPA Workshop on Innovative Cleanup Technologies, Chicago (Nov., 1999)

"Structure Analysis of Soil Humates by Liquid-State Multi-Nuclear \& Multi-Dimensional NMR", Teresa W.-M. Fan, Andrew N. Lane, and Richard M. Higashi, Annual Meeting of American Chemical Society, New Orleans (Aug., 1999).

"Heavy Metal Uptake \& Sequestration Via Plant Chelator Production", Teresa W.-M. Fan, Richard M. Higashi, and Andrew N. Lane, Annual Meeting of American Chemical Society, New Orleans (Aug., 1999).

"Metals Bioavailability and Uptake by Wheat : Cadmium and/or Humic Acids Influence", Fabienne Baraud, Teresa W-M Fan, Richard M. Higashi, Annual Meeting of American Chemical Society, New Orleans (Aug., 1999).

"Probing Interactions of Biogenic Ligands and Humic Substances That Affect The Bioavailability of Cd(II) to Plants", Richard M. Higashi, Teresa W.-M. Fan, and Fabienne Baraud, Annual Meeting of American Chemical Society, New Orleans (Aug., 1999).

"Rhizosphere Mobilization of Heavy Metals via Plant Root Exudation", Teresa W.-M. Fan, Moshe Shenker, Richard M. Higashi, David, E. Crowley, and Andrew N. Lane, Annual Meeting of American Chemical Society, Anaheim (Mar, 1999). 
"Ternary Interaction of Biogenic Ligands and Cd(II) with Humic Substances, with Implications for Metal Ion Bioavailability", Richard M. Higashi, Teresa W.-M. Fan, and Andrew N. Lane, Annual Meeting of American Chemical Society, Anaheim (Mar, 1999).

"Soil Organic Matter Structural Considerations in the Sorption and Bioavailability Of Phenanthrene", Liese F. Schultz, Richard M. Higashi, Thomas M. Young, and Teresa W.-M. Fan, Annual Meeting of American Chemical Society, Anaheim (Mar, 1999).

"Plant Rhizosphere Effects on Metal Mobilization and Transport", Teresa W.-M. Fan, Richard M. Higashi, and David E. Crowley, DOE EMSP Symposium, Chicago (July, 1998).

"Ternary Interactions Of Cd(II), Ligands, And Humic Substances - Implications For Metal Ion Bioavailability", Richard M. Higashi and Teresa W.-M. Fan, EPA, DOE, ONR, \& NSF Joint Workshop (May, 1998).

"Comprehensive Determination of Root Exudates Under Combined Fe deficiency/Cd Stress by NMR and GC-MS", T.W.-M. Fan, M. Shenker, A.N. Lane, D. Crowley, and R.M. Higashi, Bordeaux, France (April, 1998).

"Association of Biogenic Ligands with Humic Substances and Its Implications for Metal Ion Bioavailability", Richard M. Higashi, Teresa W.-M. Fan, and Andrew N. Lane, Annual Meeting of American Chemical Society, Las Vegas (Mar., 1998).

"Metal Mobilization \& Transport: Biogenic Ligands of the Plant Rhizosphere", Teresa W.-M.

Fan, Richard M. Higashi, Moshe Shenker, David Crowley, and Andrew N. Lane, DOE NABIR Workshop, Washington, DC (Jan., 1998).

"Association of Biogenic Ligands with Humic Substances and Its Implications for Metal Ion Bioavailability", Richard M. Higashi, Teresa W-M. Fan, Annual Meeting of the American Chemical Society, Las Vegas, Nevada (Sept., 1997).

"Metal Siderophore Association with Humic Substances: Implications for Metal Ion Speciation", Richard M. Higashi, Teresa W-M. Fan, and Andrew N. Lane, 3rd Int. Symposium on Speciation of Elements in Biological, Environmental, and Toxicological Sciences, Port Douglas, Australia (Sept., 1997).

b. Participate as a panel member for the "Workshop on Phytoremediaton of Inorganic Contaminants", organized by the Environmental Management division of DOE, Chicago, Nov., 1999.

Participate as Expert Panelist for the "Peer Consultation Workshop on Selenium Aquatic Toxicity and Bioaccumulation", organized by US Environmental Protection Agency, Washington, DC, May, 1998. 